\title{
Manuelle Mobilisation großflächiger Narben
}

\author{
Thomas Koller
}

Die Narbenbehandlung ist ein besonderer Schwerpunkt in der Therapie von Verbrennungspatienten. Mobilisationstechniken und Dosierung basieren auf Kenntnissen der Gewebephysiologie, Wundheilungsphasen sowie empirischem klinischem Wissen. Die Frage, welche Dosierung in welcher Wundheilungsphase zu welchem Interventionszeitpunkt angewendet werden soll, ist nicht leicht zu beantworten. Der Artikel gibt mögliche Antworten auf Grundlage klinischer Überlegungen und Erfahrungen und zeigt darauf aufbauend Befundsowie Behandlungstechniken für Verbrennungsnarben auf.

Hautverletzungen aufgrund großer Hitzeeinwirkung schädigen die Kollagenstruktur, die Matrix der Haut, die kleinen Gefäße und die Nervenendigungen. Die Fasern und Zellen, die für die Elastizität und Beweglichkeit der Haut verantwortlich sind, befinden sich meist in der Dermis. Deshalb führt ein Verlust dieser Schicht zu massiven Einschränkungen (Narbenkontrakturen). Das betroffene Areal kann unter Umständen zudem nur bedingt (je nach Schweregrad der Verbrennung) bis gar keine nozizeptiven Inputs an das ZNS weiterleiten.

Für eine wirksame manuelle Narbentherapie müssen die physiologischen und pathophysiologischen Prozesse der Wundheilung bekannt sein. Ziel ist es, funktionelle Reize in jeder Phase der Wundheilung möglichst adäquat anzuwenden.

\section{Physiologische Grundlagen der Wundheilung}

Fibroblasten sind bekanntlich sehr wichtige Zellen bei der Wundheilung. Sie bestechen mit Funktionen wie „Scannen“ des defekten Gewebes, Abbau, Synthese von verschiedenen Baustoffen (Kollagen, Elastin, Hyaluronsäure etc.) sowie Mobilität und reagieren sehr spezifisch auf mechanische Reize von außen. Dadurch nehmen Fibroblasten in der Haut und im Untergewebe eine zentrale Rolle bei der Narbenreifung ein [3][4][7].

In der Entzündungsphase (0-6 Tage) besteht ihre Hauptaufgabe darin, sich ins Wundareal fortzubewegen (Chemotaxis), das zerstörte Gewebe zu analysieren und es (unter Mithilfe der Makrophagen) abzubauen.
In der Proliferationsphase (bis zum 21. Tag) beginnen die Fibroblasten, die einzelnen Bausteine für das zu erneuernde Gewebe zu synthetisieren. Zunächst produzieren sie den unspezifischen Kollagentyp III. Dieser kann sehr schnell und einfach hergestellt werden und fungiert als provisorisches Gerüst. Er besitzt eine Turn-over-Rate von 30 Tagen und richtet sich nach mechanischen Reizen aus (॰Abb. 1):

- Wirkt in dieser Zeit kein mechanischer Reiz auf das Hautgewebe ein, legt der Fibroblast die Kollagen-TypIII-Fasern ungeordnet über den Defekt. Deshalb ist der Defekt später auch nicht funktionell belastbar.

- Wirkt in dieser Phase hingegen ein adäquater mechanischer Reiz von außen auf das defekte Hautgewebe ein, richtet der Fibroblast das Kollagen Typ III in funktioneller Richtung aus. Dies stellt eine optimale Basis für die Remodulierungsphase dar, weil das provisorische Grundgerüst bereits für das spätere Kollagen Typ I funktionell ausgerichtet ist.

- Wirkt in der Proliferations- oder Remodulierungsphase ein zu großer mechanischer Reiz auf die defekte Hautstelle ein, führt dies zu Zellwandzerstörungen auf zellulärer Ebene mit darauffolgendem Rückfall in die Entzündungsphase.

In der Remodulierungsphase (ab 21. Tag) produzieren die Fibroblasten den definitiven Kollagentyp I, welcher eine Turn-over-Rate von 300-500 Tagen (in der Haut ca. 150 Tage) aufweist und somit einen viel längeren und größeren funktionellen Reiz benötigt, um sich neu auszurichten. Er wird von den Fibroblasten exakt über das Kollagen Typ III gelegt, das zeitgleich langsam abgebaut wird. In dieser Phase ist das Gewebe also deutlich belastbarer und kann seine Funktion besser aufnehmen. Dies 
resultiert in einer besseren Verschiebbarkeit der Haut, und durch die funktionelle Ausrichtung der Matrix kann trotz fehlendem Elastin die maschendrahtartige Anordnung der Matrix eine gewisse elastische Funktion übernehmen.

Während der Proliferationsphase halten Myofibroblasten die Wundränder zusammen und verschließen diese. Diese Art der Fibroblasten besitzt vermehrt kontraktile Elemente und ist topografisch an den Wundrändern vorzufinden. Daher rührt auch die physiologische Restriktion einer Narbe. Ein zu großer mechanischer Reiz (Zug) auf die Wundränder oder -flächen generiert eine vermehrte Ansammlung von Myofibroblasten an den Wundrändern, welche mit erhöhter Restriktion reagieren. In der Folge entsteht eine vermehrte Kollagenbildung.

\section{Schmerzphysiologie in Bezug auf Verbrennungen}

Grundsätzlich gestalten sich Schmerzaussagen der Patienten in der ersten Rehabilitationsphase (Ende Proliferationsphase, Anfang Remodulierungsphase) sehr schwierig. Gründe dafür sind deren generell veränderte Körperwahrnehmung, die zum Teil zerstörten Schmerzfasern (A-delta- und C-Fasern) im primären Wundareal und die zu Beginn zu Recht hoch dosierte Schmerzmedikation. Dennoch lassen sich die subjektiven Aussagen der Patienten in einer sicherlich nicht prioritären Gewichtung mit in die Dosierung der Behandlung einbeziehen.

Was passiert nun auf Gewebeebene bezüglich der Schmerzfasern? Die A-delta-Fasern reagieren schon sehr früh und schnell auf mechanische Einflüsse. Sie melden starkes Ziehen und Druck und sind auch bei einer Schädigung aktiv. Dagegen reagieren C-Fasern vornehmlich erst bei einer Schädigung (Zellwandzerstörung). Durch die Zerstörung von Zellwänden tritt Arachidonsäure aus, die schnell zu Prostaglandin umgewandelt wird. Die C-Fasern sind sehr sensitiv gegenüber Prostaglandin und reagieren mit einer Ausschüttung von „Substanz P“. Durch diesen Vorgang beginnt die Entzündungsphase mit all ihren Entzündungszeichen (Dolor, Calor, Rubor, Tumor und Functio laesa) jeweils wieder erneut. Dies ist bei der Behandlung von Patienten mit Schwerstverbrennungen tunlichst zu vermeiden. Bei der Untersuchung und der Behandlung dürfen die Patienten bei der entsprechenden Dosierung gerade noch ein „Ziehen“ wahrnehmen (A-delta-Faser-Aktivität). Sie sollten jedoch unter allen Umständen kaum bzw. niemals anhaltende Schmerzen empfinden (C-Faser-Aktivität). Dies würde für eine erneute Entzündungsreaktion aufgrund mechanischer Überdosierung sprechen.

Eine weitere Veränderung der Schmerzwahrnehmung ist die kognitive Verarbeitung vom primären und

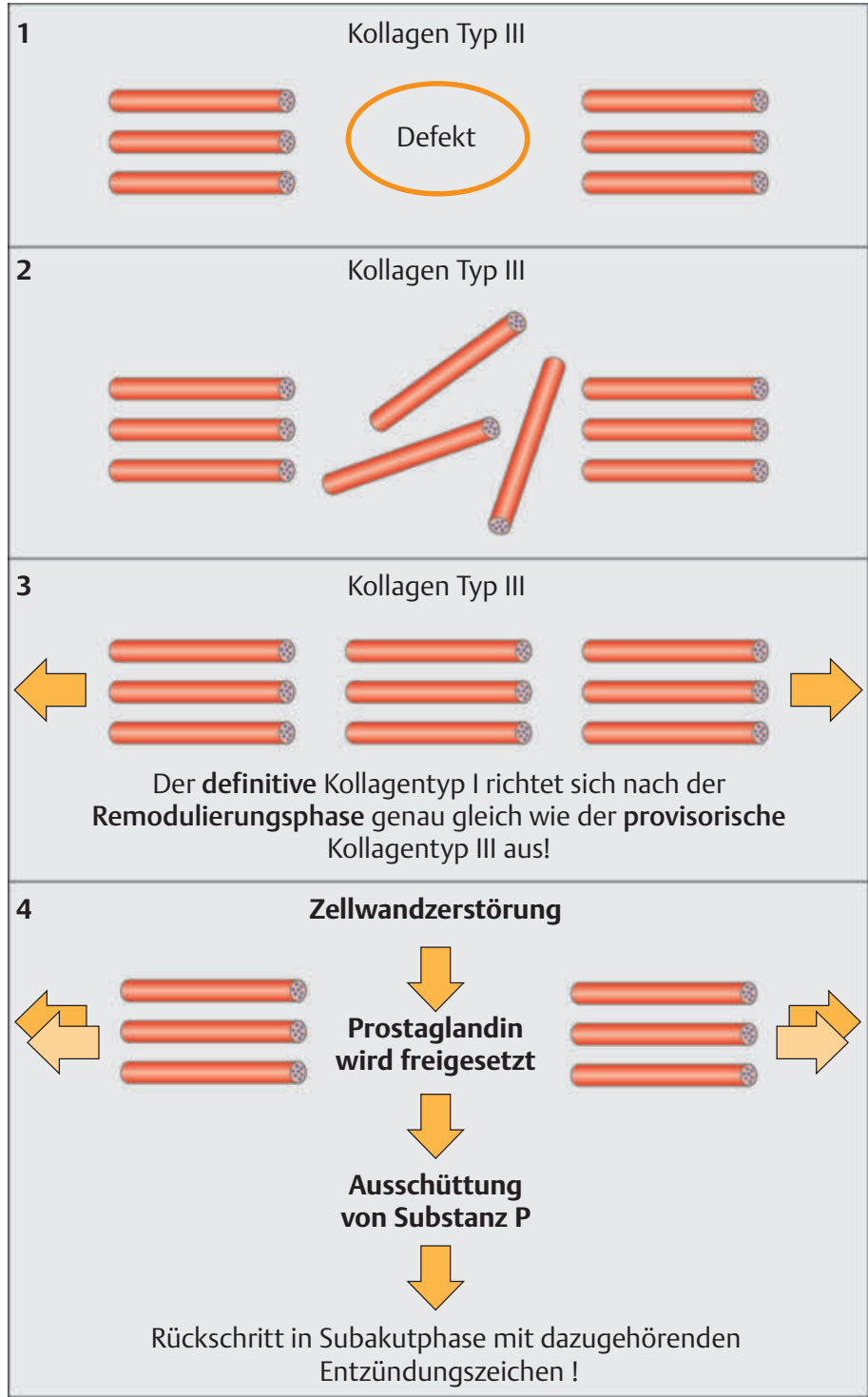

- Abb. 1 Reaktion auf zellulärer Ebene auf verschieden dosierte mechanische Reize auf die Haut. 1. Defekte Stelle im Kollagenverbund. 2. Kollagensynthese von Typ III in der Proliferationsphase ohne mechanischen Reiz von außen. 3. Kollagensynthese von Typ III in der Proliferationsphase mit adäquatem funktionellem mechanischem Reiz von außen. 4. Reaktion auf zu großen mechanischen Reiz in der Proliferationsphase (auch in der Remodulierungsphase möglich). (Grafik T. Koller; Umsetzung Thieme Verlagsgruppe)

sekundären Wundareal. Dabei wird zwischen der direkten und indirekten Schmerzleitung unterschieden.

\section{Direkte Schmerzleitung}

Physiologisch besitzt jedes Hautareal sein eigenes Abbild auf dem Kortex. Demzufolge lassen sich alle als schmerzhaft empfundenen Reize klar lokalisieren, um so eine zeitnahe motorische Schutzantwort zu generieren ( Abb. 2a). Da die Aktivität der A-delta-Faser sehr früh einsetzt, warnt sie vor erneuter zellulärer Schädigung. Theoretisch können die Patienten dann auch mehr oder weniger den Schmerz der A-delta-Faser von dem der 
C-Faser unterscheiden. Diese Schmerzqualitäten können bei der Dosierung der physiotherapeutischen Interventionen hilfreich sein.

\section{Indirekte Schmerzleitung}

Die indirekte Schmerzleitung entsteht bei jeder strukturellen Verletzungsart. In der Entzündungs- und Proliferationsphase erhält das kortikale Areal des primären Wundareals bei schwerstbrandverletzten Patienten praktisch kaum einen Input. Denn aufgrund der (durch thermische Zerstörung) zumeist fehlenden Schmerzfasern ist das primäre Wundgebiet nicht in der Lage, schmerzhafte Reize direkt an den Kortex weiterzuleiten. Somit ist es auf indirekte Informationen angewiesen (Input aus dem sekundären Wundareal). Dies birgt zwei wesentliche Nachteile in sich:

1. Bis der im primären Wundareal gesetzte Reiz mechanisch im sekundären Wundgebiet angekommen ist, droht im primären Wundgebiet bereits eine Überdosierung in Form von Zellwandschädigung. Dies bedeutet einen erneuten Rückfall in die Entzündungsphase. Die Aktivität der A-delta-Faser ist im primären Wundareal in der Entzündungs- und Proliferationsphase nicht in der Lage, vor einer erneuten zellulären Beschädigung zu warnen ( $\triangleright$ Abb. 2 b)!

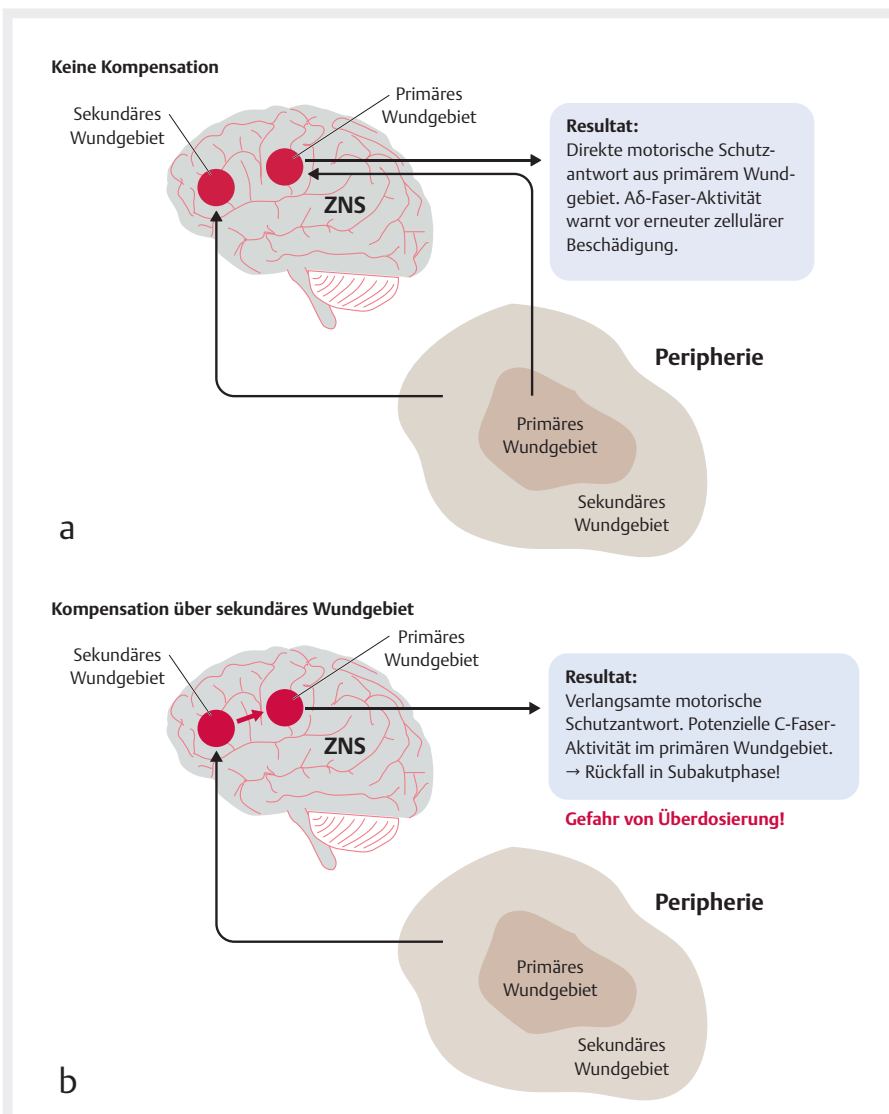

- Abb. 2 Schmerzleitung. (Quelle: Koller T. Physiotherapeutische Diagnostik. Hypothesengeleitet und klinisch relevant entscheiden. Stuttgart: Thieme; 2017) a Direkte Schmerzleitung b Indirekte Schmerzleitung.
2. Eine differenzierte Wahrnehmung ist erschwert, die motorische und/oder verbale Schutzantwort ist zusätzlich verlangsamt [8][1][5].

Diese Gegebenheiten stellen die behandelnden Physiotherapeuten vor eine große Herausforderung. Die Schmerzaussagen von schwerstverbrannten Patienten können nie ausschließlich alleine für eine adäquate Dosierung der physiotherapeutischen Interventionen verwendet werden.

\section{Dosierung mechanischer Reizsetzung}

Auf der Grundlage der Gewebe- und Schmerzphysiologie ist ein funktioneller Reiz nur über die taktile Diagnose des ersten und zweiten Bindegewebswiderstands sinnvoll.

\section{Erster und zweiter Bindegewebswiderstand}

In der Manuellen Therapie wird bei der Untersuchung von Gelenken der Widerstandsanstieg innerhalb des Bewegungsausmaßes taktil erfasst und beurteilt. Befindet sich das Gelenk in Neutralstellung (Kapsel-Band-Apparat ist entspannt), wird am Anfang in allen Bewegungsrichtungen praktisch kein Widerstand feststellbar sein. Dies wird als „neutrale Zone“ bezeichnet. Ist die neutrale Zone nicht vorhanden, spricht dies für eine Pathologie des Gelenks. Bewegt man nun weiter in eine Bewegungsrichtung, ist zunehmend ein Widerstandsanstieg spürbar. Dieser erste Widerstandsanstieg wird erster Bindegewebswiderstand genannt. Bewegt man noch weiter gegen das Ende des Bewegungsausmaßes, ist zuerst ein kontinuierlicher Widerstandsanstieg festzustellen. Dieser endet nahezu am Ende eines Bewegungsausmaßes in einem erneuten markanten Widerstandsanstieg. Dieser „endgradige“ Widerstandsanstieg wird als zweiter Bindegewebswiderstand definiert ( A Abb. 3a). Das gleiche Prinzip ist auch an der Haut zu beobachten. Wird die Haut manuell verschoben, lassen sich eine neutrale Zone sowie ein erster und zweiter Bindegewebswiderstand beobachten. Bei Verbrennungsnarben folgen die verschiedenen Zonen viel näher aufeinander und sind dementsprechend bezüglich der Dosierung viel herausfordernder ( $\mathbf{A} \mathbf{A b} \mathbf{b} . \mathbf{3 b}$ ).

\section{Überdosierung vermeiden}

Die Wundheilungsphasen dauern bei Brandverletzungen signifikant länger und das Narbengewebe ist weniger widerstandsfähig als bei linearen (chirurgischen) Narben in der gleichen Wundheilungsphase. Darüber hinaus ist es niemals homogen in sich das bedeutet, dass in Abhängigkeit von der Tiefe und Größe des Defekts, sich Narbenbereiche in unterschiedlichen Wundheilungsphasen befinden. Flächige Verbrennungsnarben sind also fragiler und bedürfen einer viel feineren Mobilisationsdosierung als lineare Narben.

Eine mögliche Überdosierung therapeutischer Interventionen und/oder die von Patienten im Alltag hervorgerufenen überdosierten Reize verschlechtern zwangsläufig die allgemeine Mobilität der defekten Hautareale. 
Zellwandzerstörungen versetzen die überbeanspruchten Areale wieder in die Entzündungsphase, und es entstehen neue offene Stellen. Diese zeugen immer von einem mechanischen Spannungsausgleich und einer vorangegangenen mechanischen Überdosierung.

Auch eine mechanische Überdosierung auf zellulärer Ebene (ohne offene Hautstellen) leitet zwangsläufig eine neue Entzündungsphase ein. Stärker vernarbte Stellen (sogenannte Narbenstränge) übertragen den mechanischen Reiz mühelos und unbeschadet, leiten ihn aber in schwächere Gewebsareale und schädigen dort unter Umständen die sich neu formierende Zellstruktur. Somit wird diese Stelle zwangsläufig wieder in einen Subakutzustand versetzt. Diese Situation ist tunlichst zu vermeiden. Es ist darauf zu achten, dass der mechanische Reiz genau an der richtigen Stelle ankommt. Dazu eignen sich folgende Dosierungsparameter:

- subjektive Aussagen der Patienten in Bezug auf Ziehen und Schmerz

- Vermeiden eines Spannungsausgleichs (Weiterlaufen der mechanischen Spannung im vernarbten Gebiet)

- Bindegewebswiderstand in taktiler Form

\section{Dosierung mittels Bindegewebswiderstand}

Es ist anzunehmen, dass manuelle Interventionen im Bereich des ersten Bindegewebswiderstands einen ausreichenden Reiz für die funktionelle Ausrichtung der extrazellulären Matrix darstellen. Klinische Erfahrungen zeigen vor allem bei schwerstbrandverletzten Patienten, dass Interventionen in der Proliferationsphase, die lediglich im Bereich des ersten Bindegewebswiderstands durchgeführt werden, deutlich weniger Rückfälle aufgrund von Überbelastungen (Zellschaden) nach sich ziehen.

In der Proliferationsphase generieren mechanisch gesetzte Reize im Bereich des ersten Bindegewebswiderstands erfahrungsgemäß lediglich eine Aktivität der A-delta-Fasern ( $\mathbf{A}$ bb. 3, grüner Bereich). Dagegen wird in der Proliferationsphase im Bereich des zweiten Bindegewebswiderstands eine potenzielle Aktivität der C-Fasern aktiviert. Grund dafür ist, dass das Kollagen Typ III in der Proliferationsphase nur begrenzt mechanischen Scherkräften und Beschleunigungen standhält. Somit besteht hier eine große Gefahr einer erneuten Zellwandbeschädigung ( $\triangleright$ Abb. 3, roter Bereich).

Während postoperative Narben bereits in der Proliferationsphase bis an den zweiten Bindegewebswiderstand dosiert werden können ( $\mathbf{A} \mathbf{b} \mathbf{b} \mathbf{4} \mathbf{4 a}$ ), sollten Therapeuten bei großflächigen Brandnarben ihre Intervention also nur bis zum ersten Anstieg des Bindegewebswiderstands dosieren ( $\triangleright \mathbf{A b b} \mathbf{4 b}$ ).

In der Remodulierungsphase ist eine Bewegung zunehmend bis zum zweiten Bindegewebswiderstand hin

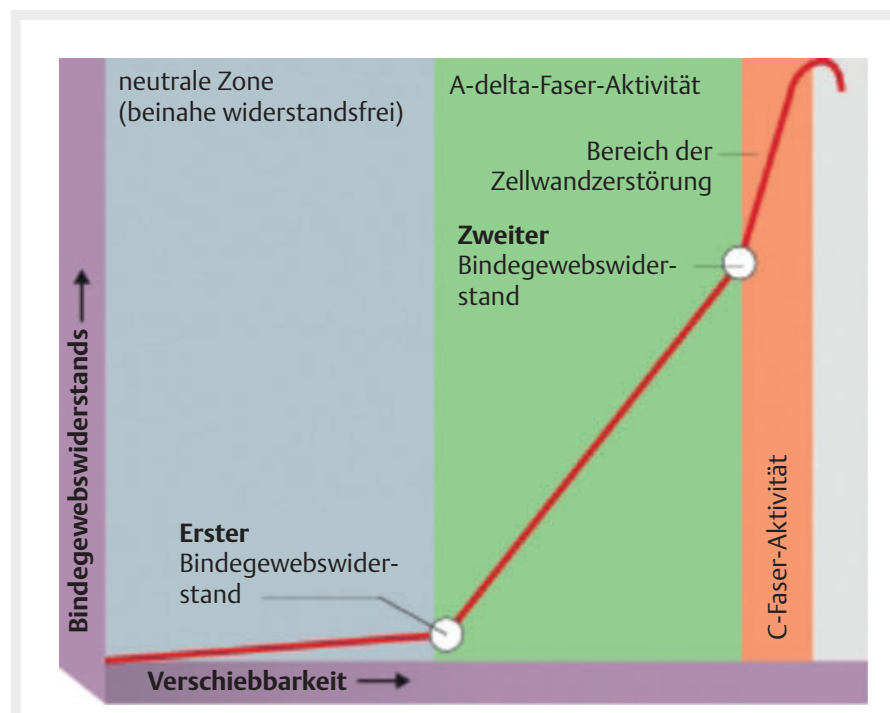

a

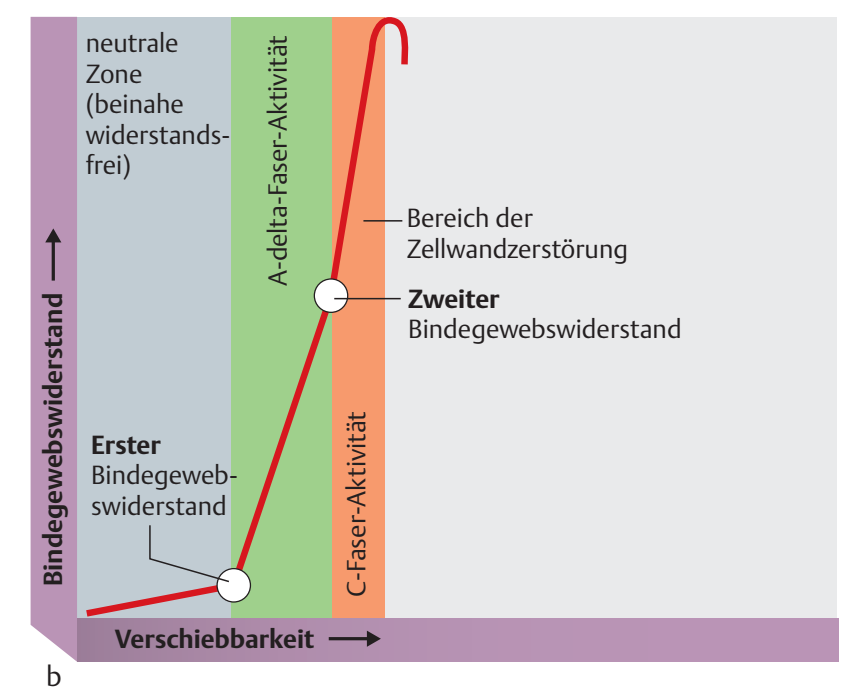

Abb. 3 Zusammenhang von Bindegewebswiderstand und Verschiebbarkeit der Haut. (Grafik T. Koller; Umsetzung Thieme Verlagsgruppe) a Normale unbeschädigte Haut. b Durch Verbrennungsnarben geschädigte Haut.

adäquat, da der definitive Kollagentyp I mechanisch wesentlich stabilere Eigenschaften aufweist [5]. Aber auch hier ist schnell eine Überdosierung möglich! Die in das neue Hautgewebe teilweise eingesprossten A-deltaFasern können im primären Wundareal in beschränktem Maße wieder ihre Funktion übernehmen. Im Falle einer Überdosierung in dieser Phase werden jedoch die inzwischen auch teilweise wieder eingesprossten C-Fasern durch Zellwandzerstörungen mit der Ausschüttung von Substanz $\mathrm{P}$ reagieren mit der Folge eines Rückfalls in die Entzündungsphase. Bei Verbrennungsnarben sollte nicht in den zweiten Bindegewebswiderstand hinein behandelt werden, vielmehr bildet dieser die Dosierungsgrenze ( Abb.4b). Postoperative Narben können hingegen zunehmend in den zweiten Bindegewebswiderstand hinein behandelt werden ( $\triangleright \mathbf{A b b} \mathbf{4 a}$ ). 


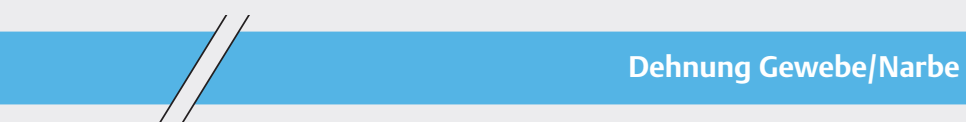

Dosierung während der Remodulierungsphase (gewebespezifisch) und später (Kollagen Typ I)

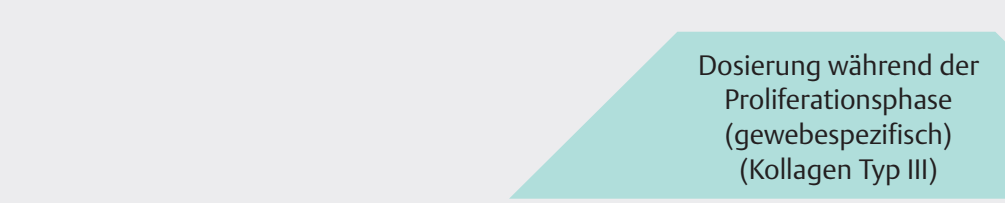

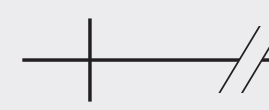

Mechanotransduktion

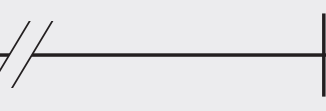

1. BGW
(R1)

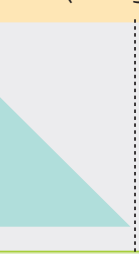

A-delta-Faseraktivität

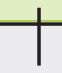

(R2)
Dosierung während der Remodulierungsphase (gewebespezifisch) und später (Kollagen Typ I)

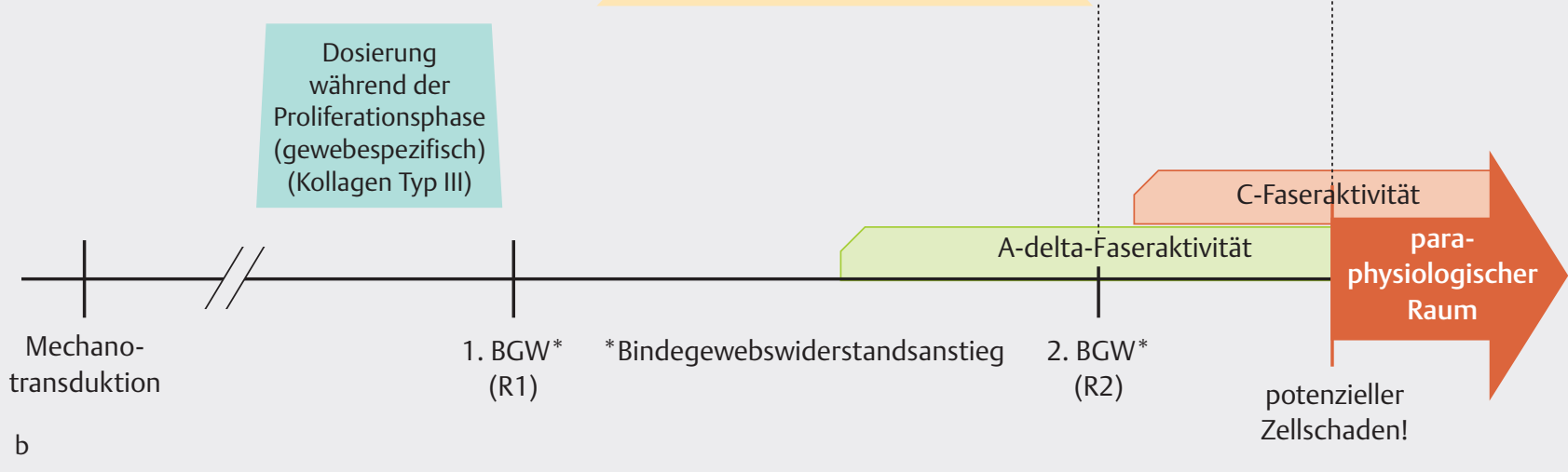

- Abb. 4 Zusammenfassende Darstellung der wundheilungsphasenadaptierten und gewebespezifischen Dosierung in der Manuellen Therapie. (Quelle: Koller T. Klinische Überlegungen bezüglich der wundheilungsphasenadaptierten und gewebespezifischen Dosierung in der Manuellen Therapie. Manuelletherapie 2019; 23: 40-46) a Bei verletztem Gewebe (posttraumatisch/postoperativ): In der Proliferationsphase bis zum zweiten Bindegewebswiderstand (BGW). In der Remodulierungsphase bis im zweiten BGW. b Bei Brandnarben: In der Proliferationsphase im Bereich des ersten BGW. In der Remodulierungsphase bis zum zweiten BGW.

\section{VORSICHT}

Die Gefahr einer Überdosierung in der Proliferationsund Remodulierungsphase scheint höher zu sein, als dass keine funktionelle Ausrichtung der Kollagenfasern stattfinden.

\section{Dosierungsempfehlungen für Brandnarben}

- in der Proliferationsphase: im Bereich der ersten wahrnehmbaren Zunahme des Bindegewebswiderstands

- in der Remodulierungsphase: Anstieg bis zum zweiten wahrnehmbaren Anstieg des Bindegewebswiderstands 
- Anwendungsdauer in beiden Phasen: in der Regel eine Minute pro Lokalisation, drei- bis fünfmal pro Therapieeinheit

\section{Befundtechniken}

Der klassische Aufbau bei der physiotherapeutischen Befundaufnahme einer Narbe oder Narbenplatte umfasst folgende Bestandteile:

1. Beurteilung der Qualität der Narbe: Hierzu gehören die Inspektion (z. B. Einziehungen, Wülste, hypertroph, kelloidartig, flach, strangartig, Färbung, offene Stellen) und die Palpation (z. B. Konsistenz, weich, dick ,,verbacken“, erwärmt).

2. Bewegungsausmaß (Range of Motion, ROM): Hier ist einerseits der globale Bewegungsausschlag zu beurteilen und andererseits, wie sich die Narbe oder Narbenplatte in Bewegung verhält: Wo entstehen potenzielle Spannungsausgleiche (mechanische Überbelastung)? Welche Narbenanteile bewegen sich spontan in die physiologische Bewegungsrichtung und welche bleiben stehen und reagieren mit Widerstand und potenzieller Überbelastung?

3. Spezifische Tests: Capillary Refill Test, Verschiebe-, Abhebe- und Verlängerungstest

Bei allen Tests soll in der Proliferationsphase nur bis zum ersten Anstieg des Bindegewebswiderstands und in der Remodulierungsphase bis zum Anstieg des zweiten Bindegebswiderstands untersucht werden.

\section{Capillary Refill Test}

Der Capillary Refill Test erlaubt Rückschlüsse auf die momentane Wundheilungsphase der Narbe. Zu Beginn der Proliferationsphase ist das Narbengewebe durch die intensive Neovaskularisation sehr gut durchblutet. Wird das Blut durch leichten mechanischen Druck aus den Kapillaren herausgedrückt, entsteht eine partiell nicht mehr durchblutete Narbenstelle; sie wird weiß. Die Kapillaren füllen sich bei Nachlassen des mechanischen Druckes natürlich wieder. Je besser diese Stelle vaskularisiert ist, desto schneller wird die lokale Narbenstelle wieder durchblutet und nimmt die Farbe des umliegenden Gewebes an. \Video. 1 Die Zeit bis zum Wiedererlangen der Umgebungsfarbe (=Revaskularisierungszeit) lässt eine Aussage über die lokale Aktivität der getesteten Stelle treffen. Gavray et al. [1] ordnen der Revaskularisierungszeit verschiedene Wundheilungsphasen zu:

\section{HINTERGRUNDWISSEN}

\section{Revaskularisierungszeit}

- < 2 Sekunden: Die Narbe befindet sich in der Proliferationsphase.
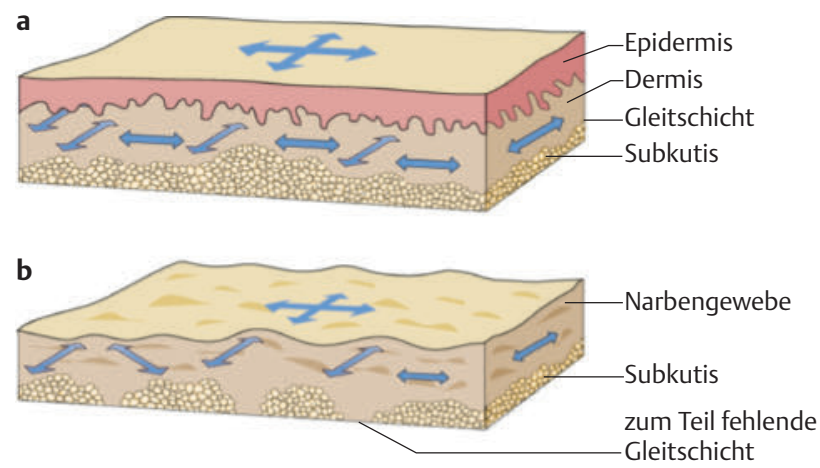

- Abb. 5 Verschiebetest. a Physiologische Gleitschicht zwischen Dermis und Subkutis. b Nur noch zum Teil vorhandene Gleitschicht. An den verklebten Stellen drohen bei Verschiebung des Gewebes eine mechanische Überbelastung und eine daraus folgende neue Entzündungsphase durch Zellwandzerstörung. (Grafik: T. Koller; Umsetzung: Thieme Verlagsgruppe)

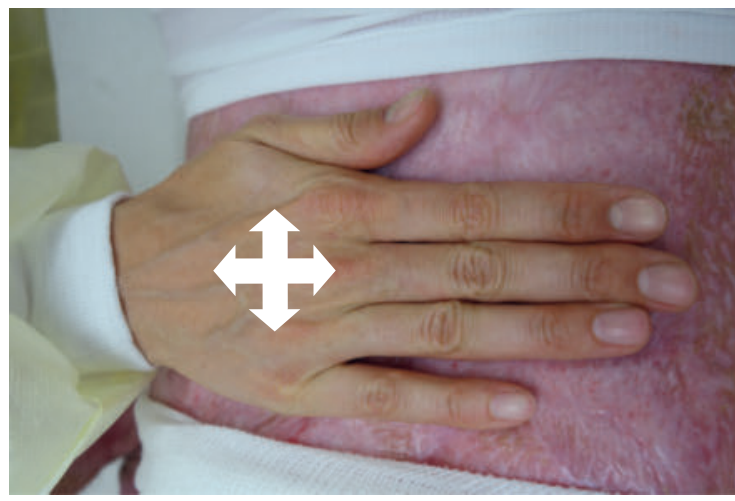

Abb. 6 Der Verschiebetest dient der Erfassung der Mobilität des Narbengewebes in verschiedene Richtungen. (Quelle: T. Koller)

\section{CAPILLARY REFILL TEST}
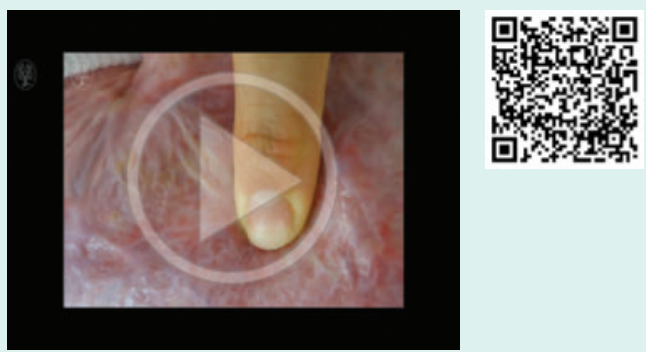

- Video Dieser Test bestimmt die grundlegende Dosierung der folgenden Testung (Verschiebe-, Abhebe-, Verlängerungstest) und sollte daher immer zuerst ausgeführt werden 


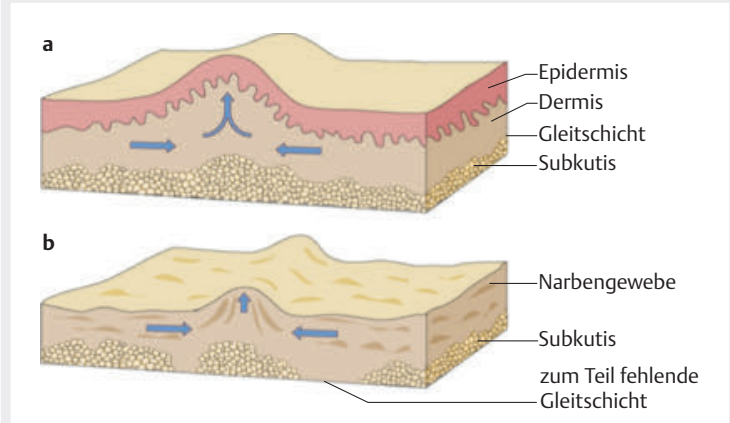

Abb. 7 Abhebetest. a Physiologische Gleitschicht zwischen Dermis und Subkutis. b Nur noch zum Teil vorhandene Gleitschicht. An den verklebten Stellen drohen beim Abheben des Gewebes eine mechanische Überbelastung und eine daraus folgende neue Entzündungsphase durch Zellwandzerstörung. (Grafik: T. Koller; Umsetzung: Thieme Verlagsgruppe)

- 2-3 Sekunden: Die Narbe befindet sich in der Remodulierungsphase.

- > 3 Sekunden: Die Narbenreifung ist nahezu abgeschlossen.

Letzteres bedeutet nicht, dass an diesen Narben funktionell keine Veränderung mehr erzielt werden kann, es gibt lediglich Auskunft über die Stabilität des Gewebes (Kollagen Typ I). Genau dieser stabile Kollagentyp I benötigt auch einen viel länger andauernden Reiz (Turnover: 150-500 Tage), bis der Fibroblast ihn funktionell ausrichten kann [8].

\section{Verschiebetest}

Das Ziel des Verschiebetests ist die Erfassung der Mobilität des Narbengewebes in verschiedene Richtungen, vor allem im Bereich der funktionell relevanten Stellen.

Bei intakter Haut befindet sich die physiologische Gleitschicht zwischen Dermis und Subkutis ( $\mathbf{A b b}$. 5a), wobei die Subkutis in sich schon eine große Mobilität aufweist (lockerer Gewebeverband; [8]). Bei einer Narbenplatte ist je nach Schwere und Tiefe der Verletzung die physiologische Gleitschicht unterbrochen, und die Dermis ist grundsätzlich Gewebe mit niedrigerer Qualität (eigentliches Narbengewebe: vornehmlich Kollagen Typ I; - Abb.5b). Beim Verschiebetest sind diese Verklebungen (Restriktionen oder Adhäsionen) in Form eines frühen und starken Widerstandsanstiegs zu spüren.

Bei der praktischen Ausführung ist ein flächiges Einsinken ins Gewebe wichtig, um einen guten Kontakt mit der Oberfläche aufnehmen zu können. Des Weiteren mindert dieses Einsinken ins Gewebe die „Wegrutschgefahr“ der Hand auf der Oberfläche und lässt erspüren, ob das Gewebe mit einem „Release“ oder einem

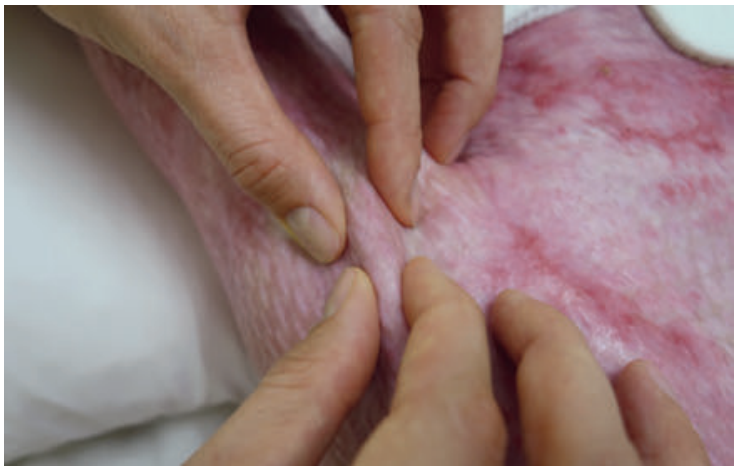

Abb. 8 Der Abhebetest erfasst die Mobilität des Narbengewebes in vertikaler Richtung. (Quelle: T. Koller)

Spannungsanstieg (nicht physiologisch) reagiert. Durch leichtes Verschieben der Hand lässt sich die Mobilität des Narbengewebes beurteilen ( $\mathbf{A b b} \mathbf{6} \mathbf{6})$.

\section{Abhebetest}

Das Ziel des Abhebetests ist die Erfassung der Mobilität des Narbengewebes in vertikaler Richtung, vor allem im Bereich der funktionell relevanten Stellen. Die Abhebbarkeit setzt sich einerseits aus der Verschiebbarkeit und andererseits aus der Dichte des Gewebes zusammen. Die Dichte (Hyperkollagenose) wird mit konstanter Kompression behandelt. Durch diese Kompression entsteht eine Hypoxie im Narbengewebe und eine daraus resultierende Apoptose. Der Abhebetest wird vornehmlich zu Beginn der Remodulierungsphase eingesetzt, da er das Gewebe mechanisch stark beansprucht.

Bei intakter Haut lässt die physiologische Gleitschicht eine dreidimensionale Mobilität zu ( $\mathbf{A} \mathbf{b} \mathbf{b}$. 7a), was zur funktionellen Abhebbarkeit der Haut führt [8]. Wie beim Verschiebetest sind hier, durch die veränderte Gleitschicht ( $\mathbf{A} \mathbf{A b} \mathbf{b} . \mathbf{7 b})$, Verklebungen in Form eines frühen und starken Widerstandsanstiegs zu spüren.

Bei der praktischen Ausführung ist ein flächiges Einsinken mit Daumen und Zeigefinger ins Gewebe wichtig, um einen guten Kontakt mit der Oberfläche aufnehmen zu können. Durch leichtes Zusammenschieben von Daumen und Zeigefinger entsteht eine Hautfalte. Je schwerer sich eine Hautfalte bilden lässt, desto dichter ist das Narbengewebe. Anschließend wird anhand einer leichten Supinationsbewegung beider Unterarme die Abhebbarkeit der Hautfalte beurteilt ( $>$ Abb. 8).

\section{Verlängerungstest}

Das Ziel des Verlängerungstests ist die Erfassung der Dehnbarkeit (Entfernung zweier Punkte) des Narbengewebes in verschiedene Richtungen. Bei intakter Haut realisiert die Dermis in sich eine gewisse Dehnfähigkeit durch Elastineinlagerungen ( $\triangleright$ Abb.9a); [8]. Die physiologische Gleitschicht unterstützt dies zusätzlich. Diese Eigenschaft ist vor allem über artikuläre Gelenke mit viel 
Bewegungsausmaß von größter Bedeutung. Bei einer Narbenplatte ist zusätzlich zur Unterbrechung der Gleitschicht ( Abb.9b) die Dehnbarkeit des Gewebes aufgrund des fast gänzlichen Fehlens von Elastin herabgesetzt [8].

Bei der praktischen Durchführung ist wie bei den anderen Tests ein flächiges Einsinken ins Gewebe wichtig. Durch leichtes Verschieben beider Hände lässt sich die Verlängerungsfähigkeit des Narbengewebes beurteilen ( Abb. 10). Wichtig ist, dass sich das Narbengewebe zwischen den beiden Händen in derselben Wundheilungsphase befinden muss. Ist dies nicht der Fall, wirkt die mechanische Dehnungskraft unweigerlich in die mechanisch schwächste Stelle ein und überlastet diese. Diese schwächste Stelle reagiert mit einem Spannungsausgleich und reißt ein. Eine neue Entzündungsphase wird initiiert. Dies ist bei allen Untersuchungstests und Behandlungstechniken zu vermeiden!

\section{Manuelle Mobilisation}

Grundsätzlich kommen die Behandlungstechniken analog der Untersuchungstests zur Anwendung. Die Dosierung wird der aktuell lokal herrschenden Wundheilungsphase angepasst. Bezüglich der Dauer der funktionellen Reizsetzung (verschieben, abheben, verlängern) finden sich in der Literatur nur wenige Angaben. Eine empirische Tendenz ist trotzdem zu erkennen, und diese ergibt aus gewebephysiologischen Überlegungen auch Sinn. Die Reizgebung soll möglichst homogen über den Tag verteilt und während der Intervention nicht hoch dosiert sein [8].

\section{Bei Patienten mit großflächigen Narben- platten hat sich eine Reizsetzung von ein bis drei Minuten pro Reiz mit zwei- bis dreimaliger Wiederholung je Interven- tionssitzung bewährt. Während des Tages soll diese Interventionssitzung zwei bis fünf Mal wiederholt werden.}

Dabei können die Patienten ab einem gewissen Stadium auch teilweise eine adäquate Reizsetzung durch geeignete Übungsanordnungen selbstständig ausführen. Bei der Platzierung der Hände muss stetig die Beschaffenheit der Narbe (hinsichtlich Qualität und Wundheilungsphase) beachtet werden. Zwischen den Händen sollte ein mechanisch möglichst homogenes Areal liegen, das sich idealerweise in der gleichen Wundheilungsphase befindet. Ist dies nicht der Fall, sollte die Platzierung der Hände so erfolgen, dass sich mit einer Hand die weiterlaufende Bewegung in die fragilen Stellen abstoppen (unterbrechen, schützen oder aussparen) lässt.

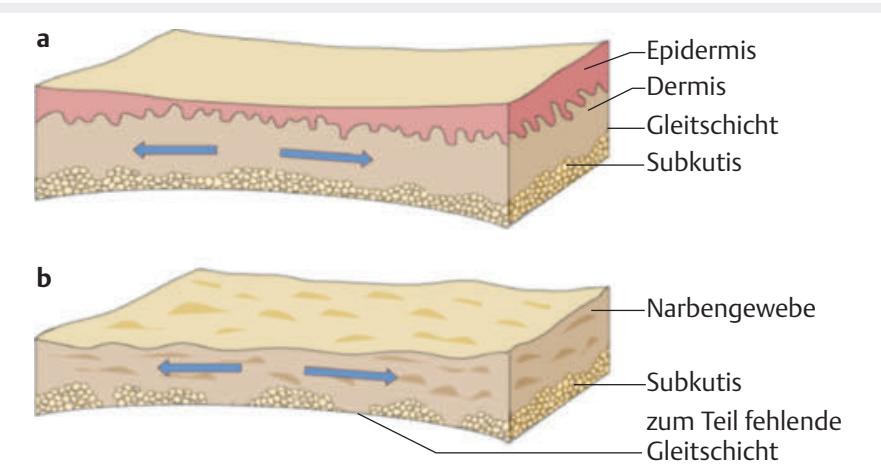

•Abb. 9 Verlängerungstest. a Physiologische Gleitschicht zwischen Dermis und Subkutis. b Nur noch zum Teil vorhandene Gleitschicht. An den verklebten Stellen drohen bei Verlängerung (Dehnen) des Gewebes eine mechanische Überbelastung und eine daraus folgende neue Entzündungsphase durch Zellwandzerstörung. Das fast gänzlich fehlende Elastin im Narbengewebe wirkt sich zusätzlich negativ auf die Dehnbarkeit aus. (Grafik: T. Koller; Umsetzung: Thieme Verlagsgruppe)

\section{Behandlungstechniken}

Verschiebe- und Abhebetechnik Das Vorgehen ist bei beiden Techniken analog der dazugehörenden Tests ( Abb.6, $\triangleright$ Abb. 8). Je nach Wundheilungsphase wird bis in den Bereich des ersten oder zweiten Anstiegs des Bindegewebswiderstands mobilisiert. Bei der Abhebetechnik kann in der Remodulierungsphase auch eine leichte Querfriktion in die Hautfalte appliziert werden (ohne auf dem Gewebe zu verrutschen).

Verlängerungstechnik Bei der Verlängerungstechnik wird zwischen der 2-Punkte- und 3-Punkte-Technik unterschieden. Die 2-Punkte-Technik kommt bei konvexen Körperstellen zur Anwendung. Dabei werden beide Hände so platziert, dass sich dazwischen die konvexe Körperstelle befindet. Die Konvexität gewährleistet, dass der mechanische Reiz homogen über die Körperstelle

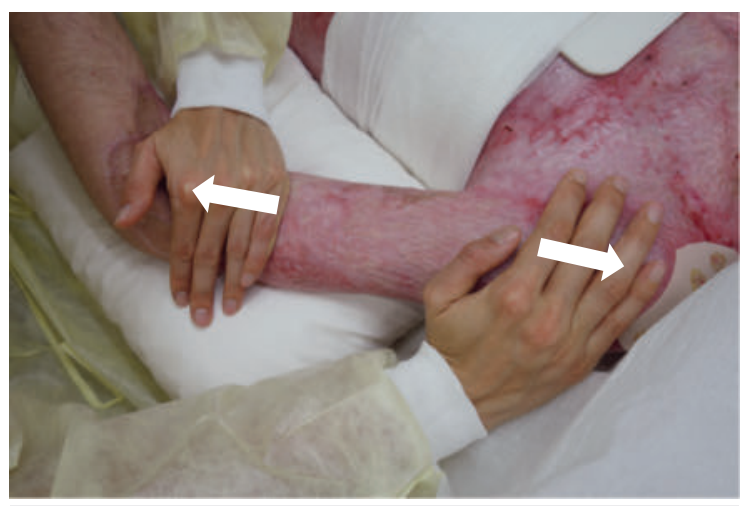

- Abb. 10 Der Verlängerungstest erfasst die Dehnbarkeit des Narbengewebes in verschiedene Richtungen. (Quelle: T. Koller) 


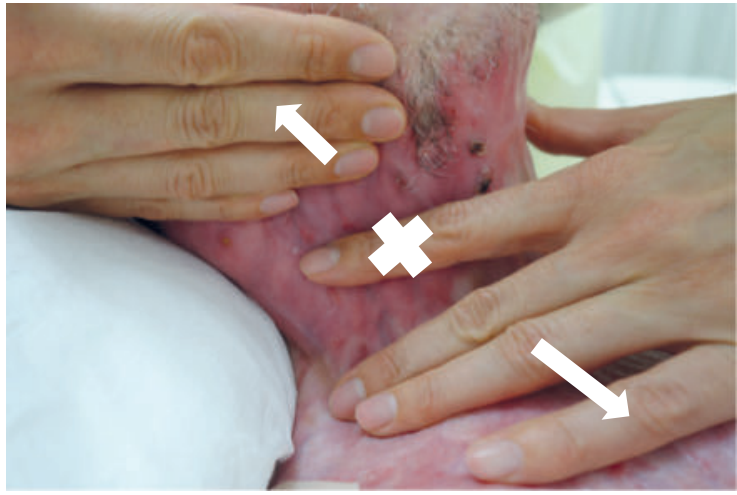

Abb. 11 3-Punkte-Technik in Verlängerung. (Quelle: T. Koller)

verteilt wird (vorausgesetzt, die Qualität der Narbe ist möglichst homogen und sie befindet sich in derselben Wundheilungsphase; $>$ Abb. 10).

Die 3-Punkte-Technik wird bei konkaven Körperstellen angewendet. Abb. 11 zeigt im Halsbereich lateral eine konkave Körperstelle. Hier würde sich bei der 2-PunkteTechnik der ganze Narbenstrang zwischen den beiden Händen abheben und den mechanischen Reiz übertragen (Weg des geringsten Widerstands direkt in eine schwächere Stelle). Somit wäre das Risiko eines Spannungsausgleiches an einer schwächeren Stelle größer. Bei der 3-Punkte-Technik werden beide Hände je an das Ende eines Narbenstrangs gesetzt, der Narbenstrang wird unter Spannung gebracht und mit einem Finger auf dem Zenit der Konkavität zum Körper hingedrückt. Dadurch fließt der mechanische Reiz gezielt in den festen Narbenstrang.

\section{Fazit}

Funktionelle Reize sind schon früh in der Proliferationsphase zu setzen, um eine funktionelle Anordnung der Kollagenfasern zu unterstützen. Um eine adäquate physiotherapeutische Narbentherapie zu applizieren, bedarf es einer taktil stetigen Kontrolle vor und während der manuellen Intervention. Sie setzt mit der Berücksichtigung des ersten und zweiten Bindegewebswiderstands in der entsprechenden Phase der Wundheilung die Grundlage für eine adäquate Dosierung. Brandnarben sind dabei vorsichtiger zu behandeln als postoperative/ posttraumatische Narben. Die subjektiven Schmerzangaben von Patienten dürfen in der Proliferationsphase nicht als einziges Instrument für die Dosierung verwendet werden. Dagegen sollten sie in der Remodulierungsphase vermehrt in die Dosierungsbestimmung mit eingebunden werden, da Patienten in dieser Phase die beiden Schmerzaktivitäten (A-delta- und C-Fasern) besser unterscheiden können.
Die Behandlungstechniken ermöglichen eine sehr spezifische und lokale Reizgebung auf das Narbengewebe. Obwohl sie zeitintensiv sind, lohnt sich dieser Aufwand: Er resultiert in einem verbesserten Bewegungsausmaß und lässt die Patienten stetig selbstständiger in ihren Aktivitäten des täglichen Lebens werden. Somit können sie im Alltag auch zunehmend autonom die funktionellen Reize setzen. Eine konsequent getragene Kompression und das Verwenden von Silikonauflagen unterstützen maßgeblich einen positiven allgemeinen Rehabilitationserfolg.

\section{Autorinnen / Autoren}

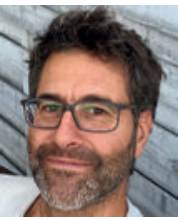

\section{Thomas Koller}

Fachexperte Physiotherapie muskuloskelettal, Orthopädische und Handchirurgische Rehabilitation, MAS msk ZFH, OMT svomp, Dipl. PT FH

\section{Korrespondenzadresse}

\section{Rehaklinik Bellikon}

Mutschellenstr. 2

5454 Bellikon

Schweiz

E-Mail: thomas.koller@rehabellikon.ch

\section{Literatur}

[1] Butler D, Moseley GM, Ween-Vermazen G et al. Schmerzen verstehen. Berlin: Springer; 2016

[2] Gavroy JR, Poveda K, Oversteyns B et al. Interêt du „test de vitropression" dans le suivi des cicatrices de brûlures à partir der 50 observations. 1995. Im Internet: www.medbc.com/annals/ review/vol_8/num_1/text/vol8n 1 p28.htm; Stand: 28.04.2017

[3] Jaudoin D, Mathieu I, Weber S et al. Physiothérapie de la cicatrice après un brûlure grave. Weiterbildungsskript. 2010. Im Inernet: kinebrul.free.fr / kinebrul / html_fr / membres / kinebrul_pro / ftp / kinebrul-pro_cicatrice.pdf; Stand: 28.04.2017

[4] Kapp H, Smola H. Regulation der Wundheilung durch Wachstumsfaktoren und Zytokine. Hartmann WundForum 2006; 1: $8-14$

[5] Koller T. Physiotherapeutische Diagnostik. Hypothesengeleitet und klinisch relevant entscheiden. Stuttgart: Thieme; 2017

[6] Koller T, Ruegg C, Gut V. Physiologische Grundlagen manueller Mobilisation von Narben und Bindegewebe sowie Dosierung bei Patienten mit großflächigen Brandverletzungen. Manuelletherapie 2016; 20: 237-241

[7] Nast A, Eming S, Fluhr J et al. Leitlinie der Deutschen Dermatologischen Gesellschaft: Therapie pathologischer Narben (hypertrophe Narben und Keloide). ICD10: L91.9. 2012. Im Inernet: www.awmf.org/uploads/tx_szleitlinien/013-0301_S1_ Pathologische_Narben_2012-04.pdf; Stand: 28.04.2017

[8] Van den Berg F. Angewandte Physiologie Bd. 1: Das Bindegewebe des Bewegungsapparates verstehen und beeinflussen. Stuttgart: Thieme; 2016 